\title{
À la recherche des gènes de la psychose maniaco-dépressive : un bilan bien déprimant
}

Dans la psychose maniaco-dépressive, l'évolution de la recherche en génétique ressemble aux phases de la maladie: aux espoirs triomphants succèdent désenchantements et incertitudes. Tout avait pourtant bien commencé, avec un locus en Xq28, dès 1969 [1], confirmé par plusieurs auteurs [2, 3], atteignant un impressionnant lod score à 7,5 dans une étude faite sur des familles israéliennes [4], mais qui, malheureusement, s'effondra par la suite [5].

En 1987, un autre locus, situé non loin du gène de la tyrosine hydroxylase, sur le bras court du chromosome 11, paraissait prometteur $(\mathrm{m} / \mathrm{s}$ $n^{\circ} 5$, vol. 3, $p$. 301). Les résultats semblaient d'autant plus cohérents que les familles analysées appartenaient au très homogène groupe de l'Ancien Ordre des Amish, descendants de Christian Fisher et d'une trentaine d'autres Européens, émigrés au début du XVIII ${ }^{\mathrm{e}}$ siècle aux USA, qui fondèrent le comté de Lancaster dans le sud-est de l'état de Pennsylvanie [6]. Mais, depuis, l'équipe de Janice Egeland, qui l'avait proposé, est revenue sur le lod score de 4,9 initialement avancé. Poursuivant l'étude épidémiologique de la maladie dans ce groupe humain génétiquement et culturellement particulier, cette même équipe vient de trouver trois autres locus, sur les chromosomes 6,13 et 15 , avec des lod scores bien plus modestes et abandonne l'hypothèse d'une transmission autosomique dominante en faveur d'une hérédité plurifactorielle de la maladie [7]. A partir de 1992, avec la pléthore des marqueurs polymorphiques balisant l'ensemble du génome, d'autres équipes tentèrent leurs chances dans de laborieuses analyses de ségrégation. Celles-ci portèrent sur d'autres isolats, en Finlande avec une autre localisation sur l'X, beaucoup plus proximale [8], au Costa-Rica où 473 marqueurs furent analysés dans une récente étude internationale sur une population de la vallée centrale appartenant à deux familles issues d'un nombre d'ascendants restreint [9]. De grandes familles isolées furent aussi étudiées [10] ainsi que des familles nucléaires, à transmission verticale parent-enfant(s), dans lesquelles les analyses de ségrégation tinrent compte du sexe du parent atteint [11]. Indiscutablement, l'étude des jumeaux (les monozygotes étant plus souvent concordants que les dizygotes), le suivi d'enfants nés d'un parent malade et ayant été adoptés (échappant donc à un climat familial pouvant être considéré comme psychologiquement pathogène), font penser qu'il existe un facteur génétique dans la psychose maniacodépressive. Sans compter les nombreux arbres généalogiques dans lesquels le mode de transmission semble autosomique dominant, surtout si, en plus des cas de troubles psychiatriques bipolaires, on retient aussi les cas de troubles unipolaires (épisodes répétés de dépression). A l'heure actuelle, quatorze locus ont été publiés [12-17] (Tableau I). Pourtant aucun n'emporte la conviction, au contraire. Après l'hypothèse d'une hétérogénéité génétique (qui comporterait au moins dix gènes diffé-

\begin{tabular}{|c|c|c|c|}
\hline \multicolumn{4}{|c|}{$\begin{array}{c}\text { Tableau I } \\
\text { LES DIFFÉRENTS LOCUS PROPOSÉS POUR D'HYPOTHÉTIQUES GÈNES } \\
\text { DE LA PSYCHOSE MANIACO-DÉPRESSIVE }\end{array}$} \\
\hline Localisation & Lod score & Année & Auteurs \\
\hline \multirow[t]{3}{*}{$X$ q 28} & - & 1969 & Reich et al. [1] \\
\hline & 13,4 & 1972 & Mendlewicz et al. [2] \\
\hline & 7,5 & 1987 & Baron et al. [4] \\
\hline $11 \mathrm{p} 15$ & 4,9 & 1987 & Egeland et al. [6] \\
\hline X q 27 & 3,1 & 1987 & Mendlewicz et al. [17] \\
\hline$X$ q $24-26$ & 3,5 & 1995 & Pekkarinen et al. [8] \\
\hline $5 q 35$ & 1,4 & 1993 & Coon et al. [13] \\
\hline 21 q 22 & 3,4 & 1994 & Craddock et al. [14] \\
\hline $18 p$ & - & 1994 & Berrettini et al. [15] \\
\hline $18 \mathrm{q} 22$ & - & 1996 & Freimer et al. [9] \\
\hline $18 q$ & $1,7-3,1$ & 1995 & Stine et al. [11] \\
\hline $16 \mathrm{p} 13$ & 2,7 & 1995 & Ewald et al. [16] \\
\hline $4 p 16$ & 4,8 & 1996 & Blackwood et al. [10] \\
\hline $6 p 24$ & 2,5 & 1996 & Ginns et al. [7] \\
\hline $13 q 13$ & 1,4 & 1996 & Ginns et al. [7] \\
\hline $15 q 11$ & 1,1 & 1996 & Ginns et al. [7] \\
\hline
\end{tabular}


rents), l'idée d'un modèle simple de transmission mendélienne est remise en cause et, dans ces conditions, est-il raisonnable de continuer à rechercher et à publier de nouveaux locus ? A défaut d'autres pistes, et même si le mode de transmission est plus complexe qu'on ne l'imaginait, il n'est pas inutile d'établir une carte d'exclusion et de vérifier si tel locus répond au mode de transmission de certaines familles, qui pourraient ainsi former des sous-groupes, dans l'attente du premier gène prédisposant qui nous éclairerait enfin sur un des mécanismes pathogéniques de cette maladie dévastatrice pour les malades (on compte $10 \%$ à $20 \%$ de suicides dans les cas non traités) et pour leur famille.

S.G.

1. Reich T, Clayton PJ, Winokar G. Family history studies: V The genetics of mania. Am J Psychiatr $1969 ; 125: 1358-69$.
2. Mendlewicz J, Fleiss JL, Fieve RR. Evidence for $\mathrm{X}$-linkage in the transmission of manic-depressive illness. J Am Med Ass 1972; 222: 1624-7.

3. Mendlewicz J, Rainer JD. Morbidity and genetic transmission in manic depressive illness. Am J Hum Genet 1974; 26 : 692-701.

4. Baron M. Genetic linkage between X-chromosome markers and bipolar affective illness. Nature 1987; 326: 289-92.

5. Baron M, Freimer NB, Risch N, Lerer B, Alexander JR, Straub RE, Asokan S, et al. Diminished support for linkage between manic depressive illness and $\mathrm{X}$ chromosome markers in three Israeli pedigrees. Nature Genet 1993; 3: 49-55.

6. Egeland JA, Bipolar affective disorder linked to DNA markers on chromosome 11. Nature 1987 ; 325: 783-7.

7. Ginns EI, Ott J, Egeland JA, Allen CR, et al. A genome-wide search for chromosomal loci linked to bipolar affective disorder in the Old Order Amish. Nature Genet 1996; 12: 431-4.

8. Pekkarinen $\mathrm{P}$, Terwilliger J, Bredbacka PE, Lonnquist J, Pettonen L. Predisposition to bipolar disorder in the genetic isolate of Eastern Finland. Psychiatr Genet 1995; 5 : S26.

9. Freimer NB, Reus VI, Escamilla MA, McInnes LA, et al. Genetic mapping using haplotype, association and linkage methods suggests a locus for severe bipolar disorder (BPI) at 18q22-q23. Nature Genet 1996; 12: 436-41.

10. Blackwood DHR, He L, Morris S, McLean A, Whitton C, et al. A locus for bipolar affective disorder on chromosome 4p. Nature Genet 1996; 12: 427-30.

11. Stine OC, Xu J, Koskela R, McMahon FJ, Gschwend M, et al. Evidence for linkage of bipolar disorder to chromosome 18 with a parent-oforigin effect. Am J Hum Genet 1995; 57 : 1384-94. 12. Risch N, Botstein D. A manic depressive history. Nature Genet 1996; 12: 351-3.

13. Coon H, Jensen S, Hoff M, Holik J, Plaetke R, Reimherr F, Wender P, Leppert M, Byerley W. A genome-wide search for genes predisposing to manic-depression, assuming autosomal dominant inheritance. Am J Hum Genet 1993; 52: 1234-49.

14. Craddock N, Owen M, Burge S, Kurian B, Thomas $\mathrm{P}$, et al. Familial cosegregation of major affective disorder and Darier's disease (keratosis follicularis). Br J Psychiatry 1994; 164: 355-8.

15. Berettini WH, Ferraro TN, Goldin LR, Weeks DE, Detera-Wadleigh S, et al. Chromosome 18 DNA markers and manic depressive illness: evidence for a susceptibility gene. Proc Natl Acad Sci USA 1994; 91 : 5918-21.

16. Ewald H, Mors O, Flint T, Koed K, Eiberg H, Kruse TA. A possible locus for manic depressive illness on chromosome 16p13. Psychiatr Gen 1995 5: 71-81.

17. Mendlewicz L, Simon P, Sevy S, Charon F, Brocas H, Legros S, Vassart G. Polymorphic DNA marker on $\mathrm{X}$ chromosome and manic depression. Lancet 1987; 1: 1230-2.
De plus en plus fort : une spermatogenèse xénogénique. Nous avons rapporté en 1994 l'exploit de l'équipe de Ralph Brinster (Philadelphie, PA, USA) qui était parvenue à repeupler des testicules de rongeurs stérilisés par un traitement au busulfan (misulban) à l'aide de spermatogonies préparées à partir d'un donneur sain ( $\mathrm{m} / \mathrm{s} n^{\circ} 12$, vol. $\left.10, p .1353\right)$. L'équipe de Ralph Brinster discutait l'intérêt d'une telle technique pour repeupler les testicules de malades stérilisés à la suite d'une chimiothérapie anticancéreuse et évoquait même la possibilité d'une thérapie génique germinale. Dans de nouvelles expériences, David Clouthier et al. ont greffé dans les testicules de souris immunodéprimées (souris Scid ou souris nude) des spermatogonies provenant de testicules de rat transgénique exprimant une construction dans laquelle le gène lac $Z$ est placé sous le contrôle du promoteur du gène de la métallo- thionéine 1 [1]. Cette construction permet de colorer en bleu les cellules de Sertoli et les cellules germinales dans lesquelles elle s'exprime. Une spermatogenèse de rat peut être observée dans certains tubes séminifères de souris, aboutissant, quoique rarement, à la formation de spermatozoïdes mûrs. A noter que les cellules de Sertoli observées ne dérivent pas du donneur, et n'expriment ainsi pas le gène lac $Z$, contrairement aux cellules germinales de rat. Au-delà du spectaculaire exploit technique que constitue cette expérience, qui n'apporte pas grand-chose de plus au point de vue théorique que les expériences antérieures, à quoi pourrait bien servir une telle utilisation de la spermatogenèse xénogénique? Les auteurs proposent que des testicules de souris immunodéprimées traitées au busulfan pourraient être utilisées pour provoquer la maturation de spermatogonies prélevées à partir de mâles de grande valeur mais stériles. Le faible rendement de la production de spermatozoïdes ne décourage pas les auteurs qui rappellent qu'une grossesse peut être provoquée par injection intracytoplasmique de spermatides [2]. Après l'ICSI (intracytoplasmic sperm injection) utilisant des gamètes immatures, les auteurs nous annoncent-ils la même opération avec des gamètes humains dont la maturation à partir d'un précurseur aurait été provoquée dans un testicule hôte animal ? Nous n'avons probablement pas fini d'entendre parler de la gymnastique procréative que pourrait autoriser la combinaison de la culture cellulaire, de la spermatogenèse xénogénique et de la fécondation par ICSI !

[1. Clouthier DE, et al. Nature 1996 ; 381: 418-21.]

[2. Testart J. médecine/sciences 1995; $11: 447-53$.] 